\title{
Resonances in a two-dimensional electron waveguide with a single delta-function scatterer
}

\author{
Daniel Boese*, Markus Lischkaṫ and L.E. Reichl \\ Center for Studies in Statistical Mechanics and Complex Systems, The University of Texas at Austin, Austin, Texas 78712
}

(August 10, 2018)

\begin{abstract}
We study the conductance properties of a straight two-dimensional electron waveguide with an $s$-like scatterer modeled by a single $\delta$-function potential with a finite number of modes. Even such a simple system exhibits interesting resonance phenomena. These resonances are explained in terms of quasi-bound states both by using a direct solution of the Schrödinger equation and by studying the Green's function of the system. Using the Green's function we calculate the survival probability as well as the power absorption and show the influence of the quasi-bound states on these two quantities.
\end{abstract}

72.20-i,72.20.Jv,03.80.+r

\section{INTRODUCTION}

Two-dimensional electron systems have been studied extensively over the past few years, both because it became feasible to construct such systems for example at a GaAs/GaAlAs interface at low temperatures and because the conductance was shown to be directly related to the transmission properties of the system. This relation is known as Landauer's formula, 13

$$
\Gamma=\frac{e^{2}}{h} T
$$

where $\Gamma$ denotes the conductance and $T$ the full transmission function (spin degrees of freedom are neglected). Eq. (11) results in a quantized conductance for straight channels. If one adds a single attractive $\delta$-function scatterer to such a straight waveguide, the combined effect of the scatterer itself and the backscattering off the walls leads to interesting phenpmena. This model was first suggested by Datta et al.t and later discussed by Bagwell et al. 16 In the following we will take a closer look at the resonance phenomena this system produces.

\section{DIRECT SOLUTION OF THE SCHRÖDINGER EQUATION}

Let us first obtain a solution of the Schrödinger equation for an electron in a two-dimensional waveguide with a $\delta$-function scatterer. The Hamiltonian is given by

$$
H=\frac{p^{2}}{2 m}+V(x, y)+V_{c}(y) .
$$

$V_{c}$ represents a confinement potential restricting the movement of the electron to the range $0<y<D$. The attractive scattering potential is given by

$$
V(x, y)=\gamma \delta(x) \delta\left(y-y_{0}\right) \quad(\gamma<0) .
$$

We can now expand any stationary solution $\psi_{E}(x, y)$ of the Schrödinger equation, $H \psi_{E}(x, y)=E \psi_{E}(x, y)$, in a Fourier series with $x$-dependent expansion coefficients using the complete set of transversal modes,

$$
\psi_{E}(x, y)=\sum_{n=1}^{\infty} c_{n}(x) \chi_{n}(y)
$$

where $\chi_{n}(y)=\sqrt{\frac{2}{D}} \sin \frac{n \pi}{D} y$. Inserting this series into the Schrödinger equation and employing orthogonality of the transversal modes we obtain a set of coupled equations,

$$
\frac{\partial^{2}}{\partial x^{2}} c_{m}(x)+k_{m}^{2} c_{m}(x)=\sum_{n} M_{m n} c_{n}(x) \delta(x)
$$

where $M_{m n}=\frac{4 m \gamma}{D \hbar^{2}} \sin \frac{m \pi y_{0}}{D} \sin \frac{n \pi y_{0}}{D}$ denote the coupling constants and $k_{n}=\sqrt{\frac{2 m}{\hbar^{2}} E-\frac{n^{2} \pi^{2}}{D^{2}}}$ the wavevector $\left(\operatorname{Im} k_{n}>0\right)$. Away from the scatterer, $x \neq 0$, the wavefunction must have the free-electron form,

$$
c_{n}(x)= \begin{cases}A_{n} e^{i k_{n} x}+B_{n} e^{-i k_{n} x} & x<0 \\ C_{n} e^{i k_{n} x}+D_{n} e^{-i k_{n} x} & x>0\end{cases}
$$

As $\psi$ must be continuous at $x=0$ and its derivative must have a finite jump there, the same conditions must hold for the expansion coefficients $c_{n}(x)$. Thus using these two conditions on Eq. (5) with the ansatz in Eq. (6) yields

$$
\begin{gathered}
A_{n}+B_{n}=C_{n}+D_{n}, \\
i k_{n}\left(C_{n}-D_{n}\right)-i k_{n}\left(A_{n}-B_{n}\right) \\
=\sum_{m} M_{n m}\left(A_{m}+B_{m}\right) .
\end{gathered}
$$

If $\psi$ is an evanescent mode, we can set $k_{n}=i \kappa_{n}$ and must require $A_{n}=0$ and $D_{n}=0$ to have a normalizable wavefunction. The transmission coefficient for propagating modes is then defined as $T_{m n}=\frac{k_{n}}{k_{m}} \frac{\left|C_{n}\right|^{2}}{\left|A_{m}\right|^{2}}$ and the total transmission function as

$$
T(E)=\sum_{\substack{m n \\ \text { (prop.) }}} T_{m n}
$$


where the sum extends over all propagating modes. The conductance is finally calculated using Eq. (11). The set of Eqs. (7) and (8) can only be solved numerically for a finite number of modes. We use the parameters $D=300 \AA$ for the width of the channel and $y_{0}=\frac{5}{12} D$ for the transversal position of the scatterer, the mass $m=0.067 m_{e}$ as the effective mass of an electron in GaAs and a scatterer strength of $\gamma=-7 \mathrm{feV} \mathrm{cm}^{2}$. With these parameters the first four energy subbands (transversal modes) open up at $E_{1}=6.24 \mathrm{meV}, E_{2}=24.94 \mathrm{meV}, E_{3}=56.12 \mathrm{meV}$ and $E_{4}=99.78 \mathrm{meV}$. Numerical results of Eq. (11) for a total number of modes $n_{t}=6$ and $n_{t}=100$ are shown in Fig. 1. It shows the expected step-like behavior at every energy subband edge. Furthermore, just before a new subband opens up, it shows an interesting dip. The waveguide blocks transmission in the lower mode just before a new higher mode opens up. It becomes completely opaque just before the second mode opens up. The drop in the conductance to the $n$-th level just before the next higher mode $n+1$ opens up is in fact a cumulative effect of all transmitting modes 1 through $n$ as can be seen from the individual transmission coefficients 0 These dips correspond to a resonance structure of the system. They can be attributed to quasi-bound states of the system with a finite lifetime, represented by a wavefunction of the forms

$$
\psi(\mathbf{x}, t)=\psi(\mathbf{x}) e^{E_{R} t / i \hbar} e^{E_{I} t / \hbar}
$$

where $E_{R}$ and $E_{I}$ denote the real and imaginary part respectively. These states are characterized by having a scattered wave even without an incident wave, i.e. $1 / T_{m n}=0$. We thus look for corresponding poles of the transmission function near each of these resonances. Figures 2 (a) and (b) show the poles corresponding to the first two dips of Fig. 1 1 confirming our assertion. The poles are always located in the analytically continued second sheet of the square root function $\left(\operatorname{Im} k_{n}<0\right)$. For the plots in Fig. 2 the square root function was chosen to have its branch cut on the negative imaginary axis which is visible as a discontinuity in the plots (It is thus the square root function with values on the first, "physical" sheet in the first through third quadrant of the complex energy plane and the square root function with values on the second sheet in the fourth quadrant that is used in Fig. 2).

\section{GREEN'S FUNCTION APPROACH}

The resonances observed in Section II can be determined from the Green's function of the system as well. We thus try to find the exact Green's function of the Hamiltonian $H$. This on the one hand allows for a direct and exact calculation of the quasi-bound state energies as poles of the Green's function (on the second sheet). On the other hand, the Green's function calculation is less compute-intensive and thus allows the calculation of both the survival probability and the power absorption of the waveguide (see Sec. IV and V). We first obtain the solution of the Green's function equation for the free waveguide with $H_{0}=\frac{p^{2}}{2 m}+V_{c}(y)$, 10

$$
\begin{aligned}
& G^{0, R / A}\left(x, y, x^{\prime}, y^{\prime}, E\right)= \\
& \quad \pm \sum_{n=1}^{\infty} \frac{2}{D} \sin \left(\frac{n \pi}{D} y\right) \sin \left(\frac{n \pi}{D} y^{\prime}\right) \frac{2 m}{\hbar^{2}} \frac{e^{ \pm i k_{n}\left|x-x^{\prime}\right|}}{2 i k_{n}} .
\end{aligned}
$$

$k_{n}$ is given as in Section II. The retarded solution $(+)$ is denoted by $G^{0, R}$, the advanced solution (-) by $G^{0, A}$. For the special potential $V(x, y)$ the integral equation for the full Green's function can be solved to yield 12.22

$$
\begin{array}{r}
G^{R / A}\left(x, y, x^{\prime}, y^{\prime}, E\right)=G^{0, R / A}\left(x, y, x^{\prime}, y^{\prime}, E\right) \\
+\frac{G^{0, R / A}\left(x, y, 0, y_{0}, E\right) G^{0, R / A}\left(0, y_{0}, x^{\prime}, y^{\prime}, E\right)}{1 / \gamma-G^{0, R / A}\left(0, y_{0}, 0, y_{0}, E\right)} .
\end{array}
$$

The transmission is solely determined by the retarded Green's function. We can thus read off the condition for the poles to be

$$
\frac{1}{\gamma}-\sum_{n} \frac{4 m}{D \hbar^{2}}\left(\sin \frac{n \pi}{D} y_{0}\right)^{2} \frac{1}{2 i k_{n}}=0
$$

It can be explicitly shown from Eq. (13) that the poles are always located on the second sheet and hence the branch cut has to be chosen as described in Section III. Evaluating Eq. (13) again numerically for $n_{t}=6$ and $n_{t}=100$ modes gives the pole locations in Table If. Apart from the quasi-bound states the attractive $\delta$-function scatterer also exhibits one single bound state. The pole locations agree exactly with the results from Section II thus confirming the interpretation of the resonance phenomena to quasi-bound states. For the discussed $\delta$-function impurity, the poles depend sensitively on the number of modes included in the computation. The typical behavior of the pole location vs. number of modes is shown in Fig. 3 for the pole just below the second subband edge. The plot demonstrates that the location of the poles and thus the conductance properties of the discussed system are not converging with an increasing number of modes. It can also be seen that they are systematically shifted to greater imaginary values, displayed in the broadening of the resonance.

The sum in Eq. (13) is in fact logarithmically diverging as each term in the sum is positive and approximately proportional to $1 / n$ for large $n$. The obtained results thus have to be always discussed for a specific number of modes. Nevertheless, the numerical results are in qualitative agreement with the behavior of an $s$-like scatterer as discussed by Kunze and Lenk 13 They thus still serve their purpose as a useful model if the $\delta$-function scatterer with a finite number of modes is interpreted as an $s$-like scatterer with finite width $D / n_{t}$ in $y$-direction instead of a true $\delta$-function scatterer.

Before proceeding let us review the resonances in the context of the last two sections. They are formed by all 
modes of the system collectively. Both propagating and evanescent modes (and therefore inter-channel coupling) are necessary. The evanescent modes are needed to build up a bound or quasi-bound state, whereas the propagating modes probe that state and therefore display the structure of the resonance. This can be seen clearly in Fig. 3 which shows the position of the pole causing the resonance just below the threshold of the second propagating mode. Keeping only two modes, $n=1$ which is propagating and $n=2$ which is extended and evanescent just below the threshold of the $n=2$ propagating mode, is necessary to create the pole and hence the resonance. This can be seen clearly in the inset of Fig. 3 where we show the conductance with only the $n=1$ propagating mode and the conductance when both the $n=1$ and $n=2$ modes are present. There is no resonance when only the $n=1$ mode is present. Thus, excluding the evanescent modes leads to unphysical kinks in the conductance near the threshold. The main contribution to any given resonance stems from the evanescent mode that is about to become propagating, because it has an infinite decay range at the band edge. The dip as well as the position of the pole are only slightly modified if more than this evanescent mode are included, but neither the dip nor the pole exist without evanescent modes. The remaining evanescent modes only alter the width and position of the resonances and hence play a minor role. It is interesting to note that when more than one scatterer is present, evanescent modes play an even more important role because they can localize coherently over more than one scatterer. The case of many scatterers has been discussed for waveguides in Ref. 1417 and for closed quantum systems in Ref. 18 .

\section{SURVIVAL PROBABILITY}

Up to now the Green's function only allowed for a more compact formulation. We now demonstrate that it will also give new insight on other quantities like the survival probability. The survival probability is defined as

$$
P(t)=\left|\left\langle\psi(t) \mid \psi_{i}\right\rangle\right|^{2}
$$

where $\left|\psi_{i}\right\rangle$ is the initial state at time $t=0$ and $|\psi(t)\rangle$ is the propagated state, which can be computed from

$$
\begin{aligned}
\psi(\mathbf{x}, t)= & \lim _{\epsilon \rightarrow 0}\left[\frac{i}{2 \pi} \int_{-\infty}^{\infty} d \mathbf{x}^{\prime} \int_{-\infty+i \epsilon}^{\infty+i \epsilon} d z\right. \\
& \left.e^{-\frac{i z t}{\hbar}} G^{R}\left(\mathbf{x}, \mathbf{x}^{\prime}, z\right) \psi_{i}\left(\mathbf{x}^{\prime}\right)\right], \quad \mathbf{x}=(x, y),
\end{aligned}
$$

where the Green's function enters explicitly into the calculation. The integration runs in the complex plane as shown in Fig. 目 (solid line). We can deform the contour and perform the integration as indicated by the dotted lines. In this case we pick up poles as well as integrations along our choice of branch cuts. For the ballistic case however, the Green's function does not have any poles and only the integrations along the cuts, performed on different sheets, remain. We now demonstrate this case explicitly, for which we write the initial state in the following form $\psi_{i}(\mathbf{x})=X(x) Y(y)$. Both $X(x)$ and $Y(y)$ are localized and do not contain an explicit $z$-dependence. For the integration along the $n$-th cut we define

$$
\begin{aligned}
& K_{n}^{0, R}\left(\mathbf{x}, \mathbf{x}^{\prime}, t\right)= \\
& \sum_{n^{\prime}} \frac{m}{\hbar^{2}} \chi_{n^{\prime}}^{*}(y) \chi_{n^{\prime}}\left(y^{\prime}\right) \int_{C_{n}} d z e^{-\frac{i z t}{\hbar}} \frac{e^{i k_{n^{\prime}}\left|x-x^{\prime}\right|}}{i k_{n^{\prime}}} .
\end{aligned}
$$

Although the integration runs on two different sheets, it is only relevant for the $n$-th square root, i.e. for $k_{n}$. Hence only one term contributes in the sum and we obtain

$$
\begin{aligned}
& K_{n}^{0, R}\left(\mathbf{x}, \mathbf{x}^{\prime}, t\right)= \\
& \frac{1}{i e^{i \frac{3}{4} \pi}} \sqrt{\frac{2 m \pi}{\hbar t}} e^{\frac{i m\left(x-x^{\prime}\right)^{2}}{2 \hbar t}} e^{\frac{i E_{n} t}{\hbar}} \chi_{n}^{*}(y) \chi_{n}\left(y^{\prime}\right) .
\end{aligned}
$$

Putting everything together we find for the survival probability of the ballistic wire

$$
\begin{aligned}
P(t)= & \left.\left|\frac{1}{i e^{i \frac{3}{4} \pi}} \sqrt{\frac{2 m \pi}{\hbar t}} \sum_{n} e^{\frac{i E_{n} t}{\hbar}}\right| \int d y Y(y) \chi_{n}(y)\right|^{2} \\
& \left.\int d x \int d x^{\prime} e^{\frac{i m\left(x-x^{\prime}\right)^{2}}{2 \hbar t}} X^{*}(x) X\left(x^{\prime}\right)\right|^{2} .
\end{aligned}
$$

In Fig. 5 we plot the survival probability for an initial state which is Gaussian-like localized in the $x$-direction and has a mode expansion for the transverse part, i.e. it couples equally strong to all channels. We distinguish between short, medium and long time behavior. For the short time behavior we observe the Zeno effect, 19 i.e. an initially non-decaying behavior. In the medium time regime we observe oscillations of all contributing channels in the decaying probability. The long time behavior is dominated by an $1 / t$ behavior.

For the case of a $\delta$-function impurity in the wire we pick up poles which contain terms like $e^{i E_{\mathrm{QBS}} t / \hbar}$ and therefore justify Eq. (10). Experimentally they can be detected by means of a Fourier transform and thus provide a tool to probe the complex spectrum. In addition we get contributions from the cuts, which do not cancel out, but are not dominating either.

\section{POWER ABSORPTION}

Another quantity of interest is the power absorption $\langle P\rangle$ of a quantum wire, which can be calculated from microscopic theory. It is related to the AC conductance $G(\omega)$ via $\langle P\rangle=E_{\mathrm{rms}}^{2} L^{2} G(\omega)$ and hence allows us to make a straight connection between quasi-bound states and dips in the conductance. 
An electric AC field of amplitude $E_{0}$ is applied to a region of length $L$, which is symmetric around the scatterer. It can be shown 20 that in linear response the power absorption of the ballistic wire behaves for small $\omega$ 's like

$$
\langle P\rangle=\frac{L^{2} e^{2} E_{0}^{2}}{2 h} \sum_{n=1}^{n_{C}}\left(\frac{\sin \left(\frac{m \omega L}{2 k_{+}^{\prime} \hbar}\right)}{\left(\frac{m \omega L}{2 k_{+}^{\prime} \hbar}\right)}\right)^{2}
$$

with $k_{+}^{\prime}=\sqrt{k_{F}^{2}-\left(\frac{n \pi}{D}\right)^{2}}$. The quasi-bound states have a profound influence on the power spectrum. Starting from the microscopic expression $P(t)=\int d \mathbf{r} \mathbf{E}(\mathbf{r}, t)$. $\langle\mathbf{j}(\mathbf{r}, t)\rangle$ one can show 21 that the quasi-bound states give Lorentzian-like contributions to the power absorption in these systems. However it is not clear whether these are positive or negative. We calculate the power absorption from

$$
\begin{aligned}
\langle P\rangle= & \frac{-\hbar \pi}{2} \int d \mathbf{r} \int d \mathbf{r}^{\prime} E(x) E\left(x^{\prime}\right) \int_{-\infty}^{\infty} d \tilde{E}_{1} \\
& \times \frac{f\left(\tilde{E}_{1}\right)-f\left(\tilde{E}_{1}+\hbar \omega\right)}{-\hbar \omega}\left\langle\tilde{E}_{1}\left|J_{x}(\mathbf{r})\right| \tilde{E}_{1}+\hbar \omega\right\rangle \\
& \times\left\langle\tilde{E}_{1}+\hbar \omega\left|J_{x}\left(\mathbf{r}^{\prime}\right)\right| \tilde{E}_{1}\right\rangle .
\end{aligned}
$$

The microscopic current elements are evaluated in a scattering state basis. Our numerical results are shown in Fig. 6. The applied field is of length $L=100 \mathrm{~nm}$, the scattering states are normalized on $L^{\prime}=4000 \mathrm{~nm}$. We clearly reproduce the analytical result for small $\omega$, which holds surprisingly well even for larger values. For the case of a $\delta$-function impurity the spectrum is still dominated by the ballistic background, which is not surprising because the impurity is strongly localized. The influence of the quasi-bound states can also be seen, however it is quite smaller than for the system discussed by $\mathrm{Na}$ and Reichl.21 In contrast to their system we see dips rather than peaks. This could be due to the fact that we excite into a quasi-bound state from a continuum state, which is why the dip shifts as a function of the Fermi energy. Na and Reichl however do excite from one quasi-bound state into another so that the signs cancel each other. In our system these resonances are too small to be observed, which can be attributed to the fact that the perturbation causing the quasi-bound state is much smaller (a $\delta$-function with a finitenumber of modes) than the large cavity in their system.23 On the other hand we can now make a clear and unambiguous connection between the dips in the DC conductance and the quasi-bound states.

\section{CONCLUSION}

We have considered a simple model for an electron waveguide. The conductance was calculated via Landauer's formula and shown to exhibit resonance phenomena. These resonances were attributed to quasi-bound states of the system by both looking at poles of the transmission coefficients and the Green's function. We have shown that the delta-function potential together with a finite number of modes models an $s$-like scatterer. Furthermore, we have demonstrated how these quasi-bound states influence the survival probability and the power absorption of the system.

\section{ACKNOWLEDGMENTS}

The authors wish to thank the Welch Foundation, Grant No. 1052 and DOE Contract No. DE-FG0394ER14405 for partial support of this work. Two of the authors (D.B., M.L.) gratefully acknowledge financial support from the German National Merits Scholarship Foundation during their stay in Austin.

* New address: Institut für Theoretische Festkörperphysik, Universität Karlsruhe, D-76128 Karlsruhe, Germany.

$\dagger$ New address: Physik-Department T30, Technische Universität München, D-85747 Garching, Germany.

${ }^{1}$ D. S. Fisher and P. A. Lee, Phys. Rev. B 23, 6851 (1981).

${ }^{2}$ A. D. Stone and A. Szafer, IBM J. Res. Develop. 32, 384 (1988).

${ }^{3}$ H. U. Baranger and A. D. Stone, Phys. Rev. B 40, 8169 (1989).

${ }^{4}$ S. Datta, M. Cahay, and M. McLennan, Phys. Rev. B 36, 5655 (1987).

${ }^{5}$ P. F. Bagwell, Phys. Rev. B 41, 10354 (1990).

${ }^{6}$ P. F. Bagwell and R. K. Lake, Phys. Rev. B 46, 15329 (1992).

7 The well potential has been chosen for numerical simplicity. In principle, a model closer to the experiment, like the harmonic oscillator potential, can be chosen as well.

${ }^{8}$ L. D. Landau and E. M. Lifshitz, Quantum Mechanics. Non-relativistic theory, 3rd ed. (A. Wheaton \& Co., Oxford, 1977).

${ }^{9}$ J. R. Taylor, Scattering Theory: The Quantum Theory on Nonrelativistic Collisions (John Wiley \& Sons, New York, 1972).

${ }^{10} \mathrm{P}$. M. Morse and H. Feshbach, Methods of Theoretical Physics (McGraw-Hill, New York, 1953), Vol. 1.

${ }^{11}$ A. Ludviksson, J. Phys. A 20, 4733 (1987).

12 P. F. Bagwell, J. Phys.: Condens. Matter 2, 6179 (1990).

${ }^{13}$ C. Kunze and R. Lenk, Solid State Comm. 84, 457 (1992).

${ }^{14}$ A. Kumar and P. F. Bagwell, Solid State Comm. 75, 949 (1990).

15 A. Kumar and P. F. Bagwell, Phys. Rev. B 439012 (1991).

16 A. Kumar and P. F. Bagwell, Phys. Rev. B 441747 (1991).

17 S. Bandyopadhyay, M. Cahay, D. Berman and B. Nayfeh, Superlattices and Microstructures 10, 327 (1991).

${ }^{18}$ L. Wendler and V. M. Fomin, Z. Phys. B 96, 373 (1995). 
19 B. Misra and E. C. G. Sudarshan, J. Math. Phys. 18, 756 (1977).

20 J. Mašek and B. Kramer, Z. Phys. B 75, 37 (1989).

${ }^{21}$ K. Na and L. E. Reichl, Phys. Rev. B 59, 13073 (1999).

${ }^{22}$ In fact, the wavefunction of the quasi-bound state of the $\delta$-function perturbation is highly localized around the scatterer.

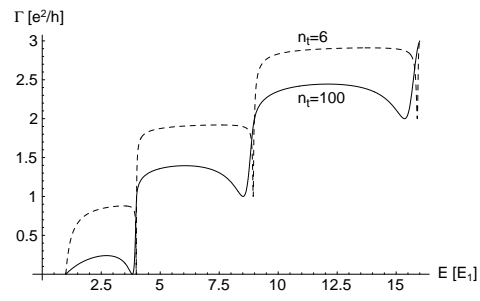

FIG. 1. Total conductance for a waveguide with a $\delta$-function scatterer of strength $\gamma=-7 \mathrm{feV} \mathrm{cm}^{2}$. The conductance goes to zero just before the second subband opens.
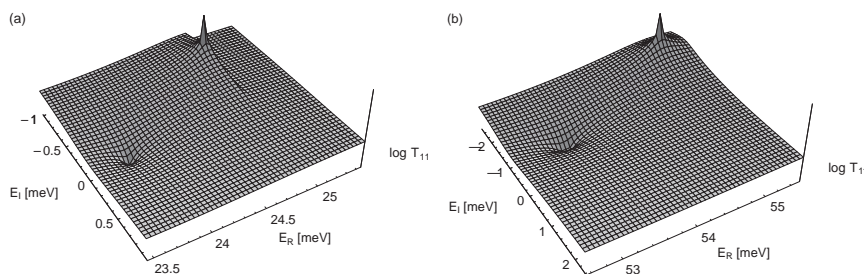

FIG. 2. Transmission poles for $\gamma=-7 \mathrm{feV} \mathrm{cm}^{2}$. The poles just below the second (a) and third (b) subband edge are shown together with the corresponding zeros which appear as dips in these logarithmic plots. The zero in (b) is off the real axis as the transmission decreases to 1 instead of 0 at this resonance (cf. Fig. 11).

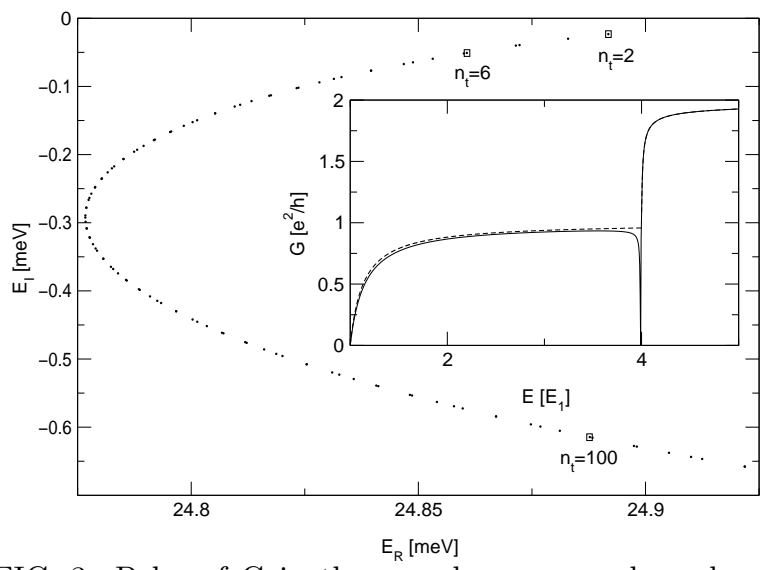

FIG. 3. Poles of $\mathrm{G}$ in the complex energy plane depending on the number of total modes $n_{t}$. The pole for $n_{t}=100$ is located at $E=24.888-0.615 i \mathrm{meV}$ and corresponds to the one shown in Fig. 2 (a). Inset: Conductance for $n_{t}=2$ (solid) and $n_{t}=$ number of propagating modes (dashed). For $E>E_{2}=4 E_{1}$ both are identical.

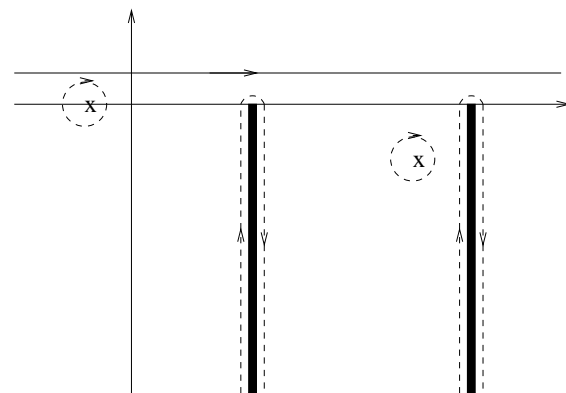

FIG. 4. Integration path for the survival probability before (solid) and after deforming the contour (dotted) in the complex $z$-plane.

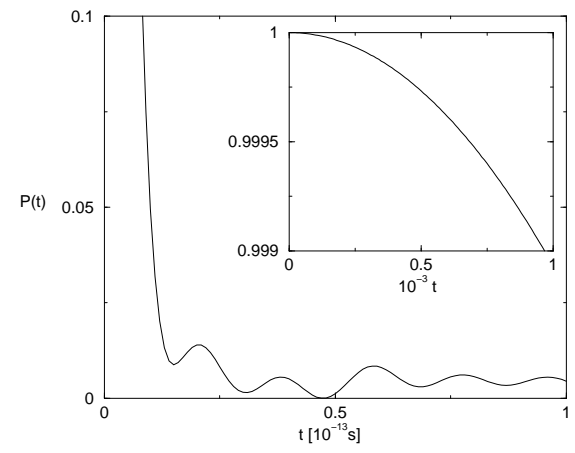

FIG. 5. The survival probability $P(t)$ as a function of time. The inset illustrates the Zeno effect for short times.

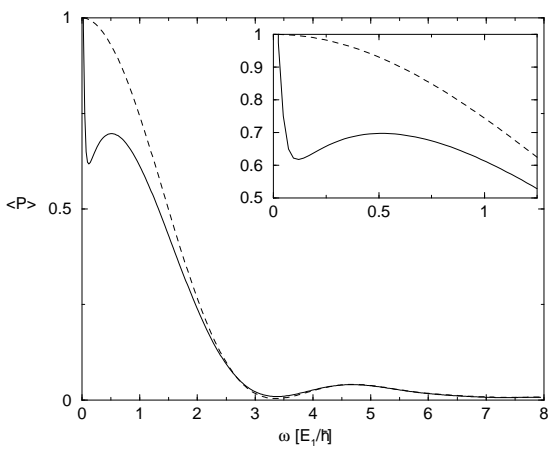

FIG. 6. Power absorption as a function of $\omega$ in units of $\frac{2 h}{e^{2} E_{0}^{2} L^{2}}$ for the ballistic (dashed) and the impurity wire (solid). The Fermi energy is close to the quasi-bound state energy.

TABLE I. Poles of $G$ for a scatterer strength of $\gamma=-7 \mathrm{feV} \mathrm{cm}^{2}$ and a total number of modes $n_{t}=6$ and $n_{t}=100$

\begin{tabular}{lll}
\hline \hline Pole & $E[\mathrm{meV}]\left(n_{t}=6\right)$ & $E[\mathrm{meV}]\left(n_{t}=100\right)$ \\
\hline 0 & 4.676 & -8.656 \\
1 & $24.861-0.051 i$ & $24.888-0.615 i$ \\
2 & $55.804-0.144 i$ & $55.117-1.961 i$ \\
3 & $99.205-0.262 i$ & $98.073-2.561 i$ \\
\hline \hline
\end{tabular}

